\title{
SCHRÖDINGER OPERATORS ON A HALF-LINE WITH INVERSE SQUARE POTENTIALS
}

\author{
HYNEK KOVAŘÍK AND FRANÇOISE TRUC
}

\begin{abstract}
We consider Schrödinger operators $H_{\alpha}$ given by equation (1.1) below. We study the asymptotic behavior of the spectral density $E\left(H_{\alpha}, \lambda\right)$ for $\lambda \rightarrow 0$ and the $L^{1} \rightarrow L^{\infty}$ dispersive estimates associated to the evolution operator $e^{-i t H_{\alpha}}$. In particular we prove that for positive values of $\alpha$, the spectral density $E\left(H_{\alpha}, \lambda\right)$ tends to zero as $\lambda \rightarrow 0$ with higher speed compared to the spectral density of Schrödinger operators with a short-range potential $V$. We then show how the long time behavior of $e^{-i t H_{\alpha}}$ depends on $\alpha$. More precisely we show that the decay rate of $e^{-i t H_{\alpha}}$ for $t \rightarrow \infty$ can be made arbitrarily large provided we choose $\alpha$ large enough and consider a suitable operator norm.
\end{abstract}

\section{Introduction}

This paper is concerned with Schrödinger operators

$$
H_{\alpha}=-\frac{d^{2}}{d x^{2}}+\frac{\alpha}{x^{2}}, \quad \alpha \geq-\frac{1}{4},
$$

in $L^{2}\left(\mathbb{R}^{+}\right)$with Dirichlet condition at $x=0$. In particular, we are interested in the dependence of various spectral properties of $H_{\alpha}$ on the parameter $\alpha$. Note that potentials of the type $\alpha / x^{2}$ have a special role, since the resulting operator $H_{\alpha}$ is scaling invariant. Moreover, it is known that the potentials which satisfy $V(x) \sim x^{-2}$ as $x \rightarrow \infty$ represent a borderline case for certain important spectral inequalities such as dispersive or Strichartz estimates, see [GVV].

It is therefore not surprising that Schrödinger operators with inverse square potentials have recently attracted certain attention; we might mention for example the heat kernel bounds obtained in [MS, MS2, or Strichartz estimates in dimension three studied in [BPST1, BPST2]. Inverse square potentials appear naturally also in connection with two-dimensional Schrödinger operators with Aharonov-Bohm-type magnetic field, see [FFFP, GK].

Main objects of our interest here are the spectral density

$$
E\left(H_{\alpha}, \lambda\right)=\frac{1}{2 \pi i} \lim _{\varepsilon \rightarrow 0+}\left(\left(H_{\alpha}-\lambda-i \varepsilon\right)^{-1}-\left(H_{\alpha}-\lambda+i \varepsilon\right)^{-1}\right), \quad \lambda>0
$$

of $H_{\alpha}$, and the unitary group $e^{-i t H_{\alpha}}$. In particular, we are going to study the asymptotic behavior of $E\left(H_{\alpha}, \lambda\right)$ for $\lambda \rightarrow 0$ and the $L^{1} \rightarrow L^{\infty}$ dispersive estimates associated to the evolution operator $e^{-i t H_{\alpha}}$. It is very well-known that the asymptotic behavior of $E\left(H_{\alpha}, \lambda\right)$ for small $\lambda$ is closely related to the asymptotic behavior of $e^{-i t H_{\alpha}}$ for large $t$. There is a huge amount of literature on this subject, see e.g. [EG, GS, JK, Mu, Sch1, Sch2, Wed1, Wed2] and references therein. We are not going to discuss this connection any further since it will not be used in our proofs. 
For general one-dimensional Schrödinger operators of the type $H_{V}=-\frac{d^{2}}{d x^{2}}+V$ the behavior of both $E\left(H_{V}, \lambda\right)$ and $e^{-i t H_{V}}$ is known provided the potential $V$ decays fast enough at infinity. In particular, if zero is a regular point of $H_{V}$, (which is the generic case), then

$$
E\left(H_{V}, \lambda\right) \sim \lambda^{\frac{1}{2}}, \quad \lambda \rightarrow 0
$$

in a suitable operator topology, see [Go, Mu, Sch2, Wed2]. Accordingly, for such short range potentials, under certain regularity conditions, Murata [Mu proved

$$
\left\|w^{-1} e^{-i t H_{V}} w^{-1}\right\|_{L^{2}(\mathbb{R}) \rightarrow L^{2}(\mathbb{R})} \leq C t^{-\frac{3}{2}} \quad \forall t>2,
$$

where $w$ is a weight function with a sufficient growth at infinity. The corresponding $L^{1} \rightarrow L^{\infty}$ was established by Schlag

$$
\left\|\rho^{-1} e^{-i t H_{V}} \rho^{-1}\right\|_{L^{1}(\mathbb{R}) \rightarrow L^{\infty}(\mathbb{R})} \leq C t^{-\frac{3}{2}} \quad \forall t>2,
$$

with $\rho(x)=(1+|x|)$, see [Sch2]. It is important to mention that the decay conditions on $V$, under which all the above results were obtained, imply that $V(x)=o\left(x^{-2}\right)$ as $|x| \rightarrow \infty$.

The goal of the present note is to show that if $V$ is of type $\alpha x^{-2}$ with $\alpha>0$, then the asymptotic relation (1.3) is no longer valid and has to replaced by a new one, and, on the other hand, the estimates (1.4) and (1.5) can be improved. In particular $E\left(H_{\alpha}, \lambda\right)$ decays faster to zero than in (1.3), see Theorem 2.1. Accordingly the decay in the dispersive estimate 1.5 can be improved provided the weight function $\rho$ grows fast enough at infinity, see Theorem 2.4. Although our results regard a family of Schrödinger operators with explicit potentials, it can be expected that similar results should hold also if $H_{\alpha}$ is perturbed by a sufficiently short-range perturbation.

It should be finally mentioned that our main results, i.e. Theorems 2.1 and 2.4, fail in the case of Schrödinger operators on the whole line due to the presence of the zero resonance.

\section{Main results}

2.1. Notation. We set $\rho(x)=1+x$ on $\mathbb{R}^{+}$. For any $s \in \mathbb{R}$ we denote

$$
L_{s}^{2}\left(\mathbb{R}^{+}\right)=\left\{u:\left\|\rho^{s} u\right\|_{L^{2}\left(\mathbb{R}^{+}\right)}<\infty\right\}, \quad\|u\|_{0, s}:=\left\|\rho^{s} u\right\|_{L^{2}\left(\mathbb{R}^{+}\right)} .
$$

Let $\mathscr{B}\left(s, s^{\prime}\right)$ be the space of bounded linear operators from $L_{s}^{2}\left(\mathbb{R}^{+}\right)$to $L_{s^{\prime}}^{2}\left(\mathbb{R}^{+}\right)$and let $\|\cdot\|_{\mathscr{B}\left(s, s^{\prime}\right)}$ denote the corresponding operator norm. Finally, we put

$$
\nu=\sqrt{1 / 4+\alpha} .
$$

We have

Theorem 2.1. Let $\alpha>-1 / 4$. Then for any $\varepsilon>0$ and any $s \geq \nu+1+\varepsilon$ it holds

$$
E\left(H_{\alpha}, \lambda\right)=E_{0} \lambda^{\nu}+\mathcal{O}\left(\lambda^{\nu+\varepsilon}\right) \quad \lambda \rightarrow 0+
$$

in $\mathscr{B}(s,-s)$, where $E_{0}$ is the integral operator in $L^{2}\left(\mathbb{R}^{+}\right)$with the kernel

$$
E_{0}(x, y)=\frac{(x y)^{\nu+\frac{1}{2}}}{2^{\nu} \Gamma^{2}(\nu+1)} .
$$


Remark 2.2. Equation (2.2) shows that for positive values of $\alpha$ the density $E\left(H_{\alpha}, \lambda\right)$ is of lesser order than in the case of a short-range potential, see equation (1.3).

Remark 2.3. For a throughout discussion of threshold expansion of resolvents of onedimensional operators with short-range potentials we refer to [JN]. Asymptotic behaviour of Schrödinger groups generated by operators with inverse square decay on conical manifolds was studied in $\mathrm{Wa}$ in the setting of weighted $L^{2}$-spaces.

Theorem 2.4. Let $\alpha \geq-1 / 4$. Then for any $s \in[0, \nu+1 / 2]$ there exists a constant $C(\alpha, s)$ such that

$$
\left\|\rho^{-s} e^{-i t H_{\alpha}} \rho^{-s}\right\|_{L^{1}\left(\mathbb{R}^{+}\right) \rightarrow L^{\infty}\left(\mathbb{R}^{+}\right)} \leq C(\alpha, s) t^{-\frac{1}{2}-s} \quad \forall t>0 .
$$

Remark 2.5. For $-1 / 4<\alpha \leq 0$ the dispersive estimate (2.3) can be derived from [FFFP, Thm.1.11] by considering the restriction of inequality [FFFP, Eq.(1.29)] to radial functions. On the other hand, the result for $\alpha>0$, namely the faster decay of $e^{-i t H_{\alpha}}$ in $t$ is new. The maximal decay rate $t^{-1-\nu}$, achieved by the choice $s=\nu+1 / 2$, should be compared with the $t^{-\frac{3}{2}}$ decay rate in the estimate (1.5).

Remark 2.6. Note also that in the border-line case $\alpha=-1 / 4$, which means $\nu=0$, Theorem 2.4 with the choice $s=1 / 2$ gives the decay rate $t^{-1}$, which is the decay rate of the free evolution $e^{i t \Delta}$ in dimension two. This is not surprising since the operator $-\frac{d^{2}}{d x^{2}}-\frac{1}{4 x^{2}}$ in $L^{2}\left(\mathbb{R}^{+}\right)$with Dirichlet boundary condition at zero is unitarily equivalent, by means of the unitary mapping $f(x) \mapsto \sqrt{x} f(x)$, to the Laplacian $-\Delta$ in $L^{2}\left(\mathbb{R}^{2}\right)$ restricted to radial functions.

An immediate consequence of Theorem 2.4 is the following

Corollary 2.7. Let $\alpha \geq-1 / 4$. Then for any $s \in[0, \nu+1 / 2]$ and any $\beta>s+1 / 2$ there exists a constant $C_{2}$, depending only on $\alpha, \beta$ and $s$, such that

$$
\left\|\rho^{-\beta} e^{-i t H_{\alpha}} \rho^{-\beta}\right\|_{L^{2}\left(\mathbb{R}^{+}\right) \rightarrow L^{2}\left(\mathbb{R}^{+}\right)} \leq C_{2} t^{-\frac{1}{2}-s} \quad \forall t>0 .
$$

Proof. Let $u \in L_{\beta}^{2}\left(\mathbb{R}^{+}\right)$and let $f=\rho^{s} u$. Then by the Cauchy-Schwarz inequality $f \in L^{1}\left(\mathbb{R}^{+}\right)$and

$$
\|f\|_{L^{1}\left(\mathbb{R}^{+}\right)}^{2}=\left(\int_{0}^{\infty} \rho^{s} \rho^{-\beta} \rho^{\beta} u d x\right)^{2} \leq C_{1}\left\|\rho^{\beta} u\right\|_{L^{2}\left(\mathbb{R}^{+}\right)}^{2},
$$

where we have used the fact that $\beta>s+1 / 2$. Hence from Theorem 2.4 and 2.5 we obtain

$$
\begin{aligned}
\left\|\rho^{-\beta} e^{-i t H_{\alpha}} u\right\|_{L^{2}\left(\mathbb{R}^{+}\right)}^{2} & =\int_{0}^{\infty} \rho(x)^{2 s-2 \beta}\left|\rho^{-s} e^{-i t H_{\alpha}} \rho^{-s} f\right|^{2} d x \\
& \leq C^{2}(\alpha, s) t^{-1-2 s}\|f\|_{L^{1}\left(\mathbb{R}^{+}\right)}^{2} \\
& \leq C^{2}(\alpha, s) C_{1} t^{-1-2 s}\left\|\rho^{\beta} u\right\|_{L^{2}\left(\mathbb{R}^{+}\right)}^{2} .
\end{aligned}
$$

This proves 2.4.

Inequality 2.4 should be compared with the estimate 1.4 valid for short-range potentials. 


\section{Proofs}

3.1. Proof of Theorem 2.1. For simplicity we shall drop the index $\alpha$ in the sequel and write $E(\lambda)$ instead of $E(\alpha, \lambda)$. We also use the notation

$$
R(\lambda, x, y)=\lim _{\varepsilon \rightarrow 0+}\left(H_{\alpha}-\lambda-i \varepsilon\right)^{-1}(x, y) .
$$

We first study the solutions $u \in L^{2}\left(\mathbb{R}^{+}\right)$of the generalized eigenvalue equation

$$
-u^{\prime \prime}-\frac{\alpha}{x^{2}}=\lambda u \text {. }
$$

After setting $u(x)=\sqrt{x} \psi(\sqrt{\lambda} x)$, equation (3.1) writes

$$
z^{2} \psi^{\prime \prime}-x+z \psi^{\prime}+\left(z^{2}-\nu^{2}\right) \psi=0,
$$

with $z=\sqrt{\lambda} x$. The latter is a Bessel equation of the first kind, see [AS, Sec.9.1]. We now find two solutions $u_{1}, u_{2}$ of (3.1) which satisfy $u_{1}(0)=0$ and $u_{2} \in L^{2}(1, \infty)$ for $\operatorname{Im} \lambda>0$. Since

$$
\left|J_{\nu}(z)+i Y_{\nu}(z)\right| \sim \sqrt{\frac{2}{z \pi}}\left|e^{i z}\right|, \quad|z| \rightarrow \infty, \quad \operatorname{Im} z>0
$$

see [AS, Eqs.9.1.3, 9.2.3], and

$$
J_{\nu}(z)=\frac{(z / 2)^{\nu}}{\Gamma(\nu+1)}+o\left(z^{\nu}\right) \quad z \rightarrow 0 .
$$

by [AS, Eq.9.1.7], the sought solutions $u_{1}$ and $u_{2}$ take the form

$$
\begin{aligned}
& u_{1}(x)=\sqrt{x} J_{\nu}(\sqrt{\lambda} x) \\
& u_{2}(x)=\sqrt{x}\left(J_{\nu}(\sqrt{\lambda} x)+i Y_{\nu}(\sqrt{\lambda} x)\right) .
\end{aligned}
$$

Hence by the theory of Sturm-Liouville problems we obtain the resolvent kernel

$$
\begin{array}{ll}
R(\lambda, x, y)=\frac{i \pi}{2} \sqrt{x y} J_{\nu}(\sqrt{\lambda} x)\left(J_{\nu}(\sqrt{\lambda} y)+i Y_{\nu}(\sqrt{\lambda} y)\right) & (x \leq y) \\
R(\lambda, x, y)=\frac{i \pi}{2} \sqrt{x y} J_{\nu}(\sqrt{\lambda} y)\left(J_{\nu}(\sqrt{\lambda} x)+i Y_{\nu}(\sqrt{\lambda} x)\right) & (x \geq y)
\end{array}
$$

The Stone formula 1.2 then implies that

$$
E(\lambda, x, y)=\frac{1}{\pi} \operatorname{Im} R(\lambda, x, y)=\frac{1}{2} \sqrt{x y} J_{\nu}(\sqrt{\lambda} x) J_{\nu}(\sqrt{\lambda} y) .
$$

From (3.3) we now easily verify that

$$
\lim _{\lambda \rightarrow 0+} \lambda^{-\nu} E(\lambda, x, y)=\frac{(x y)^{\nu+\frac{1}{2}}}{2^{\nu} \Gamma^{2}(\nu+1)}=E_{0}(x, y) .
$$

Let us define the rest term $E_{1}(\lambda)$ as the integral operator in $L^{2}\left(\mathbb{R}^{+}\right)$with the kernel given by

$$
E_{1}(\lambda, x, y)=E(\lambda, x, y)-E_{0}(x, y) \lambda^{\nu} .
$$

To prove Theorem 2.1 we need the following

Lemma 3.1. For any $\varepsilon>0$ and any $s>\nu+1+\varepsilon$ we have

$$
\left\|E_{1}(\lambda)\right\|_{\mathscr{B}(s,-s)}=\mathcal{O}\left(\lambda^{\nu+\varepsilon}\right) \quad \lambda \rightarrow 0+.
$$

Proof.- 
We will use the fact that

$$
\left\|E_{1}(\lambda)\right\|_{\mathscr{B}(s,-s)}=\left\|\rho^{-s} E_{1}(\lambda) \rho^{-s}\right\|_{L^{2}\left(\mathbb{R}^{+}\right) \rightarrow L^{2}\left(\mathbb{R}^{+}\right)} .
$$

From 3.10 we get that

$$
\rho^{-s} \lambda^{-\nu} E(\lambda) \rho^{-s}=\rho^{-s} E_{0} \rho^{-s}+\rho^{-s} \lambda^{-\nu} E_{1}(\lambda) \rho^{-s} .
$$

Note that the operator $E_{0}$ is Hilbert-Schmidt in $\mathscr{B}(s,-s)$. This follows from the identity (3.12) applied to $E_{0}$. Hence by applying the Taylor formula to the operator $\rho^{-s} \lambda^{-\nu} E(\lambda) \rho^{-s}$ at $\lambda=0$ we find that the claim of the Lemma will follow if we show that

$$
\begin{aligned}
\left\|\rho^{-s} \partial_{\lambda}\left(\lambda^{-\nu} E(\lambda)\right) \rho^{-s}\right\|_{H S\left(\mathbb{R}^{+}\right)} & =\left\|\rho^{-s} \partial_{\lambda}\left(\lambda^{-\nu} E_{1}(\lambda)\right) \rho^{-s}\right\|_{H S\left(\mathbb{R}^{+}\right)} \\
& =\mathcal{O}\left(\lambda^{-1+\varepsilon}\right) \quad \lambda \rightarrow 0,
\end{aligned}
$$

where $\|\cdot\|_{H S\left(\mathbb{R}^{+}\right)}$denotes the Hilbert-Schmidt norm in $L^{2}\left(\mathbb{R}^{+}\right)$. Using the recurrence relations for the derivatives of $J_{\nu}$ :

$$
\begin{aligned}
& J_{\nu}^{\prime}(z)=-J_{\nu+1}(z)+\frac{\nu}{z} J_{\nu}(z) \\
& J_{\nu}^{\prime}(z)=J_{\nu+1}(z)-\frac{\nu}{z} J_{\nu}(z),
\end{aligned}
$$

see [AS, Eq.9.1.27], we get from (3.8)

$$
\begin{aligned}
\partial_{\lambda}\left(\lambda^{-\nu} E(\lambda, x, y)\right)=-\frac{\lambda^{-\nu-1 / 2}}{4} \sqrt{x y}[ & \left(x J_{\nu+1}(\sqrt{\lambda} x) J_{\nu}(\sqrt{\lambda} y)\right. \\
& \left.+y J_{\nu+1}(\sqrt{\lambda} y) J_{\nu}(\sqrt{\lambda} x)\right] .
\end{aligned}
$$

Hence by the Cauchy-Schwarz inequality

$$
\begin{aligned}
\left\|\rho^{-s} \partial_{\lambda}\left(\lambda^{-\nu} E(\lambda)\right) \rho^{-s}\right\|_{H S\left(\mathbb{R}^{+}\right)}^{2}= \\
\quad=\int_{0}^{\infty} \int_{0}^{\infty}\left|\partial_{\lambda}\left(\lambda^{-\nu} E(\lambda, x, y)\right)\right|^{2} \rho(x)^{-2 s} \rho(y)^{-2 s} d x d y \\
\quad \leq C \lambda^{-1-2 \nu} \mathcal{I}(\lambda) \mathcal{J}(\lambda)
\end{aligned}
$$

where

$$
\begin{aligned}
& \mathcal{I}(\lambda)=\int_{0}^{\infty} x^{3} J_{\nu+1}^{2}(\sqrt{\lambda} x)(1+x)^{-2 s} d x \\
& \mathcal{J}(\lambda)=\int_{0}^{\infty} y J_{\nu}^{2}(\sqrt{\lambda} y)(1+y)^{-2 s} d y
\end{aligned}
$$

and $C$ is a constant independent of $\lambda$. To estimate the last two integrals we will need a point-wise estimate on the Bessel function $J_{\nu}$. From the integral representation

$$
J_{\nu}(z)=\frac{2\left(\frac{z}{2}\right)^{\nu}}{\sqrt{\pi} \Gamma\left(\nu+\frac{1}{2}\right)} \int_{0}^{1}\left(1-t^{2}\right)^{\nu-\frac{1}{2}} \cos (z t) d t,
$$

see [AS, Eq.9.1.20], it follows that $\left|J_{\nu}(z)\right| \leq C_{\nu} z^{\nu}$ for all $z>0$ and $\nu>0$. On the other hand, by [AS, Eq.9.1.20] we have $\left|J_{\nu}(z)\right| \leq 1$ for all $z>0$ 
and $\nu \geq 0$. A combination of these two upper bounds then implies that for any $-1 / 2 \leq \mu \leq \nu$ there exists a constant $C(\mu, \nu)$ such that

$$
\left|J_{\nu}(z)\right| \leq C(\mu, \nu) z^{\mu} \quad \forall z>0, \quad \forall \mu \in\left[-\frac{1}{2}, \nu\right] .
$$

Using (3.17) with $\mu=\nu-1+2 \varepsilon$ in $\mathcal{I}(\lambda)$ and with $\mu=\nu$ in $\mathcal{J}(\lambda)$ together with the fact that $s \geq \nu+1+\varepsilon$, we find

$$
\mathcal{I}(\lambda)=\mathcal{O}\left(\lambda^{\nu-1+2 \varepsilon}\right), \quad \mathcal{J}(\lambda)=\mathcal{O}\left(\lambda^{\nu}\right) \quad \lambda \rightarrow 0 .
$$

In view of (3.16) this implies (3.14) and therefore completes the proof.

Theorem 2.1 now follows from 3.10 and 3.11 .

3.2. Proof of Theorem 2.4. We will prove Theorem 2.4 by estimating the integral kernel of the operator $e^{-i t H_{\alpha}}$. To provide a formula for the integral kernel, we will follow [Ko, Sec.5], where the formula for the integral of the heat semi-group $e^{-t H_{\alpha}}$ was established, see also GK]. Equation (3.8) in combination with the Weyl-Titchmarsh-Kodaira Theorem, cf. [DSch, Chap.13], shows that the operator $H_{\alpha}$ is unitarily equivalent to a multiplication operator, namely we have

$$
\mathcal{U}_{\nu} H_{\alpha} \mathcal{U}_{\nu}^{-1} f(p)=p f(p), \quad f \in \mathcal{U}_{\nu}\left(D\left(H_{\alpha}\right)\right),
$$

where $D\left(H_{\alpha}\right)$ denotes the operator domain of $H_{\alpha}$ and the mappings $\mathcal{U}_{\nu}, \mathcal{U}_{\nu}^{-1}: L^{2}\left(\mathbb{R}_{+}\right) \rightarrow$ $L^{2}\left(\mathbb{R}_{+}\right)$are given by

$$
\begin{aligned}
\left(\mathcal{U}_{\nu} g\right)(p) & =\int_{0}^{\infty} g(x) \sqrt{x} J_{\nu}(x \sqrt{p}) d x \\
\left(\mathcal{U}_{\nu}^{-1} f\right)(x) & =\frac{1}{2} \int_{0}^{\infty} f(p) \sqrt{x} J_{\nu}(x \sqrt{p}) d p
\end{aligned}
$$

The mapping $\mathcal{U}_{\nu}$ and $\mathcal{U}_{\nu}^{-1}$ define unitary operators on $L^{2}\left(\mathbb{R}_{+}\right)$. Let $g \in C_{0}^{\infty}\left(\mathbb{R}^{+}\right)$. By $[\mathrm{T}$, Thm.3.1]

$$
e^{-i t H_{\alpha}} g=\lim _{\varepsilon \rightarrow 0+} e^{-(\varepsilon+i t) H_{\alpha}} g .
$$

In view of 3.18 we thus get

$$
\begin{aligned}
\lim _{\varepsilon \rightarrow 0+}\left(e^{-(\varepsilon+i t) H_{\alpha}} g\right)(r)=\lim _{\varepsilon \rightarrow 0+}\left(\mathcal{U}_{\nu}^{-1} e^{-(\varepsilon+i t) p} \mathcal{U}_{\nu} g\right)(x) \\
\quad=\lim _{\varepsilon \rightarrow 0+} \frac{1}{2} \int_{0}^{\infty} \sqrt{x y} \int_{0}^{\infty} e^{-(\varepsilon+i t) p} J_{\nu}(x \sqrt{p}) J_{\nu}(y \sqrt{p}) d p g(y) d y \\
\quad=\lim _{\varepsilon \rightarrow 0+} \frac{1}{2(\varepsilon+i t)} \int_{0}^{\infty} \sqrt{x y} I_{\nu}\left(\frac{x y}{2(\varepsilon+i t)}\right) e^{-\frac{x^{2}+y^{2}}{4(\varepsilon+i t)}} g(y) d y .
\end{aligned}
$$

where we have used [Erd, Eq.4.14(39)] to calculate the integral with respect to $p$. Moreover, from [AS, Eq.9.6.18] it follows that the function

$$
I_{\nu}\left(\frac{x y}{2(\varepsilon+i t)}\right) e^{-\frac{x^{2}+y^{2}}{4(\varepsilon+i t)}}
$$


is bounded on every compact interval uniformly with respect to $\varepsilon>0$. Since the support of $g$ is compact, we can use the dominated theorem and interchange the limit and integration in (3.21). Taking the limit $\varepsilon \rightarrow 0$ and using the identity $I_{\nu}(i z)=e^{-i \nu \pi / 2} J_{\nu}(z)$, see [AS, Eq.9.6.3], we obtain

$$
\left(e^{-i t H_{\alpha}} g\right)(x)=\frac{1}{2 i t} \int_{0}^{\infty} \sqrt{x y} J_{\nu}\left(\frac{x y}{2 t}\right) e^{-\frac{x^{2}+y^{2}}{4 i t}} e^{-\frac{i \nu \pi}{2}} g(y) d y .
$$

Now we apply the upper bound (3.17) with $\mu=s-1 / 2 \in[-1 / 2, \nu]$ and $z=\frac{x y}{2 t}$. This yields

$$
\sup _{x, y \in \mathbb{R}^{+}}\left|\rho(x)^{-s} \sqrt{x y} J_{\nu}\left(\frac{x y}{2 t}\right) \rho(y)^{-s}\right|<C(\alpha, s) t^{\frac{1}{2}-s} .
$$

The last equations now imply that

$$
\left\|\rho^{-s} e^{-i t H_{\alpha}} \rho^{-s} f\right\|_{L^{\infty}\left(\mathbb{R}^{+}\right)} \leq C(\alpha, s) t^{-\frac{1}{2}-s}\|f\|_{L^{1}\left(\mathbb{R}^{+}\right)}
$$

for all $f \in L^{1}\left(\mathbb{R}^{+}\right)$. This proves inequality 2.3 .

\section{Acknowledgements}

H.K. would like to thank the Institut Fourier in Grenoble for the warm hospitality extended to him during his stay. The work of H.K. has been partially supported by the MIUR-PRIN'2010-11 grant for the project "Calcolo della variation". Both authors thank the referee whose remarks and comments helped them improve the original version of the text.

\section{REFERENCES}

[AS] M. Abramowitz and I. Stegun, Handbook of mathematical functions. National Bureau of Standards, 1964.

[BPST1] N. Burq, F. Planchon, J. Stalker, S. Tahvildar-Zadeh: Strichartz estimates for the wave and Schrödinger equations with the inverse-square potential. J. Funct. Anal. 203 (2003) 519-549.

[BPST2] N. Burq, F. Planchon, J. Stalker, S. Tahvildar-Zadeh: Strichartz estimates for the wave and Schrödinger equations with potentials of critical decay. Indiana Univ. Math. J. 53 (2004) 16651680 .

[DSch] N. Dunford, J.T. Schwartz, Linear Operators, Part II. New York 1988.

[Erd] A. Erdelyi: Tables of integral transforms, Vol. 2. McGraw-Hill, New York 1954.

[EG] M. B. Erdogan, W. R. Green: A weighted dispersive estimate for Schrödinger operators in dimension two. Comm. Math. Phys. 319 (2013) 791-811.

[FFFP] L. Fanelli, V. Felli, M. A. Fontelos, A. Primo: Time decay of scaling critical electromagnetic Schrödinger flows. Comm. Math. Phys. 324 (2013) 1033-1067.

[Go] M. Goldberg: Transport in the one-dimensional Schrödinger equation. Proc. Amer. Math. Soc. 135 (2007) 3171-3179.

[GS] M. Goldberg and W. Schlag: Dispersive estimates for Schrödinger operators in dimensions one and three. Comm. Math. Phys. vol. 251 (2004) 157-178.

[GVV] M. Goldberg, L. Vega, N Visciglia: Counterexamples of Strichartz inequalities for Schrödinger equations with repulsive potentials. Int. Math Res. Not. 2006, article ID 13927 (2006).

[GK] G. Grillo, H. Kovařík: Weighted dispersive estimates for two-dimensional Schrödinger operators with Aharonov-Bohm magnetic field. arxiv: 1210.7648v (2012). 
[JK] A. Jensen, T. Kato: Spectral properties of Schrödinger operators and time-decay of the wave functions. Duke Math. J. 46 (1979) 583-611.

[JN] A. Jensen, G. Nenciu: Schrödinger operators on the half line: Resolvent expansions and the Fermi golden rule at thresholds. Proc. Indian Acad. Sci. (Math. Sci. 116 (2006) 375-392.

[Ko] H. Kovařík: Heat kernels of two-dimensional magnetic Schrödinger and Pauli operators. Calc. Var. Partial Differential Equations 44 (2012) 351-374.

[MS] P.D. Milman, Yu. A. Semenov: Heat kernel bounds and desingularizing weights. J. Funct. Anal. 202 (2003) 1-24.

[MS2] P.D. Milman, Yu. A. Semenov: Global heat kernel bounds via desingularizing weights. J. Funct. Anal. 212 (2004) 373-398.

[Mu M. Murata: Asymptotic expansions in time for solutions of Schrödinger-type equations. J. Funct. Anal. 49 (1982), 10-56.

[Sch1] W. Schlag: Dispersive estimates for Schrödinger operators in dimension two. Comm. Math. Phys. 257 (2005), 87-117.

[Sch2] W. Schlag: Dispersive estimates for Schrödinger operators: a survey. Mathematical aspects of nonlinear dispersive equations, 255-285, Ann. of Math. Stud., 163, Princeton Univ. Press, Princeton, NJ, 2007.

[T] G. Teschl: Mathematical Methods in Quantum Mechanics With Applications to Schrödinger Operators. American Mathematical Society Providence, Rhode Island, 2009.

[Wa] X.P. Wang: Asymptotic expansion in time of the Schrdingier group on conical manifolds. Ann. Inst. Fourier 56 (2006) 1903-1945.

[Wed1] R. Weder: $L^{p}-L^{p^{\prime}}$ Estimates for the Schrödinger Equation on the Line and Inverse Scattering for the Nonlinear Schrödinger Equation with a Potential. J. Funct. Anal. 170 (2000), 37-68.

[Wed2] R. Weder: The $L^{p}-L^{p}$ estimate for the Schrödinger equation on the half-line. J. Math. Anal. Appl. 281 (2003) 233-243.

Hynek Kovařík, DiCATAM, Sezione di Matematica, Università degli Studi di Brescia, ITALY

E-mail address: hynek.kovarik@ing.unibs.it

Françoise Truc, Unité miXte de Recherche CNRS-UJF 5582, BP 74, 38402-Saint Martin D'Hères Cedex (France)

E-mail address: francoise.truc@ujf-grenoble.fr 